Neu diagnostiziertes multiples Myelom

\title{
Verlängertes Überleben mit Zoledronsäure
}

Patienten mit neu diagnostiziertem multiplem Myelom können von der zusätzlichen Gabe von Zoledronsäure zur Standardchemotherapie profitieren. In der MRC Myeloma IX Study wurde u.a. ein verlängertes Gesamtüberleben gezeigt. Laut Gareth J. Morgan, London/Großbritannien, unterstützen die Studienergebnisse die bereits zuvor in Studien gezeigte Antitumorwirkung von Zoledronsäure.

In der Phase-III-Studie erhielten 1.960 Patienten mit neu diagnostiziertem multiplem Myelom zusätzlich zu einer Standardchemotherapie Clodronsäure (1.600mg/d p.o.; $\mathrm{n}=979$ ) oder Zoledronsäure (4mg i.v. alle 3-4 Wochen; $\mathrm{n}=981$ ). Die Standardchemotherapie war je nach Alter und Gesundheitszustand der Patienten eine Hochdosischemotherapie mit anschließender Stammzelltransplantation oder eine konventionell dosierte Chemotherapie.
Beim progressionsfreien und Gesamtüberleben, beides primäre Endpunkte der Studie, zeigte sich nach einem medianen Follow-up von 3,7 Jahren ein signifikanter Vorteil zugunsten der zusätzlichen Gabe von Zoledronsäure $(p=0,0179$ bzw. $p=0,0118)$. Das Gesamtüberleben wurde um median 5,5 Monate verlängert (Abb.). Der Effekt auf das Gesamtüberleben war dabei unabhängig vom Effekt auf Skelettkomplikationen (Skeletal-related Events [SRE]). SRE waren unter Zoledronsäure seltener als unter Clodronsäure $(p=0,0004)$.

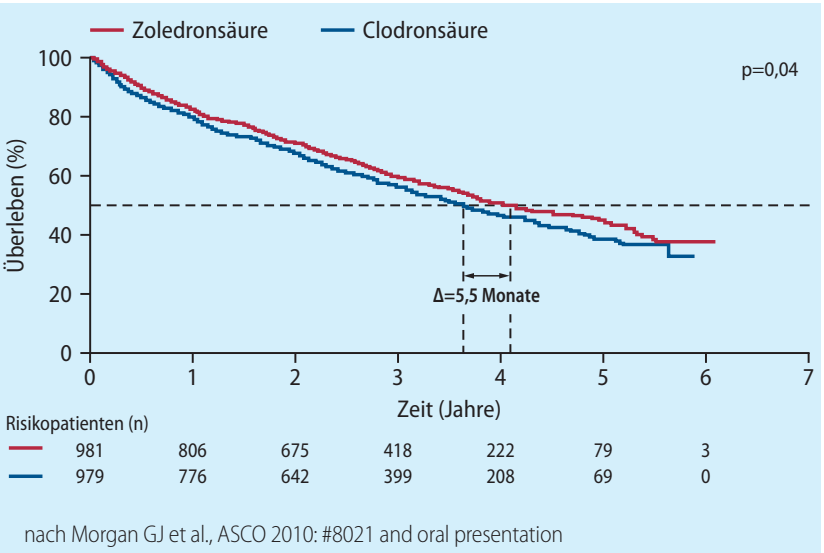

Gesamtüberleben - zusätzliche Gabe von Clodronsäure versus Zoledronsäure in der MRC (Medical Research Council) Myeloma IX Study

In der Diskussion der Studiendaten wertete Morgan die Ergebnisse zum Vergleich der beiden Bisphosphonate als klinisch signifikant. Zoledronsäure ist für Morgan der neue Therapiestandard für Patienten mit multiplem Myelom. AM

\section{Metastasiertes Mammakarzinom}

\section{Capecitabin-Monotherapie im Vergleich die bessere Wahl}

Die Monotherapie mit dem oralen Fluoropyrimidin Capecitabin hat sich als Standardtherapie des metastasierten Mammakarzinoms etabliert. Im Vergleich mit anderen Chemotherapien weist die Monotherapie mit Capecitabin Vorteile auf.

Patientinnen mit metastasiertem Mammakarzinom, die für eine intensive Chemotherapie nicht geeignet sind, können von einer First-line-Monotherapie mit Capecitabin (Xeloda ${ }^{\circledR}$ ) profitieren mehr als vom klassischen CMF-Schema (Cyclophosphamid p.o. plus i.v. Methotrexat und 5-Fluorouracil). Das konnten Stockler et al. in einer randomsierten Phase-III-Studie mit mehr als 300 Patientinnen zeigen (Stockler MR et al., 2007, J Clin Oncol 25 (Suppl): \#1031). Die Tumoransprechrate war unter Capecitabin und CMF vergleichbar ( $21 \%$ vs. $18 \%$; $p=0,8)$, ebenso das mediane progressionsfreie Überleben ( 6 Monate vs. 7 Monate; $p=0,2$ ). Beim medianen Gesamtüberleben zeigte sich jedoch ein signifikanter Unterschied zugunsten der Monotherapie mit Capecitabin (22 Monate vs. 18 Monate; Hazard-Ratio 0,72; $p=0,02$ ). Die Patientinnen - etwa $40 \%$ waren jünger als 60 Jahre - konnten länger mit Capecitabin behandelt werden. Die Capecitabin-Monotherapie war insgesamt besser verträglich und führte seltener zu Therapieabbrüchen. Auch bestimmte Aspekte der Lebensqualität, insbesondere der Haarerhalt, waren unter Capecitabin besser als unter CMF.
Wirksamkeit nach Anthrazyklin- und/oder Taxan-haltiger Vortherapie

Ein verlängertes medianes Gesamtüberleben unter Capecitabin zeigte sich auch in der retrospektiven Analyse von Verma et al. im Vergleich zu Vinorelbin nach Anthrazyklin- und Taxan-haltiger Vortherapie (188 Tage vs. 102 Tage; $p<0,0001$; Verma S et al., 2007, J Clin Oncol 30: 297-302). In die Analyse gingen die Daten von Patientinnen aus drei kanadischen Krebszentren ein, die nach Anthrazyklin- und Taxan-haltiger Vortherapie Capecitabin $(n=68)$, Vinorelbin $(n=45)$ oder eine Sequenztherapie mit beiden Wirkstoffen $(n=27)$ erhielten. Die mediane Behandlungszeit war mit Capecitabin länger als mit Vinorelbin (129 Tage vs. 64 Tage). Patientinnen, die eine Sequenztherapie erhielten, überlebten im Median 390 Tage.

Die Wirksamkeit der Capecitabin-Monotherapie nach Versagen einer Therapie mit Anthrazyklinen und/oder Taxanen konnte zuvor auch bereits in zwei zulassungsrelevanten Phase-Il-Studien von Blum et al. gezeigt werden (Blum JL et al., 1999, J Clin Oncol 17: 485-493; Blum JL et al., 2001, Cancer 92: 1759-1768).

Quelle: Informationen der Roche Pharma AG 